\title{
Productivity links morphology, symbiont specificity and bleaching in the evolution of Caribbean octocoral symbioses
}

\author{
David M Baker ${ }^{1,2,3}$, Christopher J Freeman ${ }^{4,5}$, Nancy Knowlton ${ }^{2}$, Robert W Thacker ${ }^{4}$, \\ Kiho Kim ${ }^{6}$ and Marilyn L Fogel ${ }^{3,7}$ \\ ${ }^{1}$ School of Biological Sciences, Department of Earth Science, and Swire Institute of Marine Science, The \\ University of Hong Kong, Hong Kong, PRC; ${ }^{2}$ Smithsonian Marine Science Network, National Museum of \\ Natural History, Washington, DC, USA; ${ }^{3}$ Geophysical Laboratory, Carnegie Institution of Washington, \\ Washington, DC, USA; ${ }^{4}$ Department of Biology, University of Alabama at Birmingham, Birmingham, AL, USA; \\ ${ }^{5}$ Smithsonian Marine Station, Fort Pierce, FL, USA and ${ }^{6}$ Department of Environmental Science, American \\ University, Washington, DC, USA
}

\begin{abstract}
Many cnidarians host endosymbiotic dinoflagellates from the genus Symbiodinium. It is generally assumed that the symbiosis is mutualistic, where the host benefits from symbiont photosynthesis while providing protection and photosynthetic substrates. Diverse assemblages of symbiotic gorgonian octocorals can be found in hard bottom communities throughout the Caribbean. While current research has focused on the phylo- and population genetics of gorgonian symbiont types and their photophysiology, relatively less work has focused on biogeochemical benefits conferred to the host and how these benefits vary across host species. Here we examine this symbiosis among 11 gorgonian species collected in Bocas del Toro, Panama. By coupling light and dark bottle incubations (P/R) with ${ }^{13} \mathrm{C}$-bicarbonate tracers, we quantified the link between holobiont oxygen metabolism with carbon assimilation and translocation from symbiont to host. Our data show that P/R varied among species, and was correlated with colony morphology and polyp size. Sea fans and sea plumes were net autotrophs $(P / R>1.5)$, while nine species of sea rods were net heterotrophs with most below compensation $(P / R<1.0) .{ }^{13} \mathrm{C}$ assimilation corroborated the $P / R$ results, and maximum $\delta^{13} C_{\text {host }}$ values were strongly correlated with polyp size, indicating higher productivity by colonies with high polyp SA:V. A survey of gorgonian-Symbiodinium associations revealed that productive species maintain specialized, obligate symbioses and are more resistant to coral bleaching, whereas generalist and facultative associations are common among sea rods that have higher bleaching sensitivities. Overall, productivity and polyp size had strong phylogenetic signals with carbon fixation and polyp size showing evidence of trait covariance. The ISME Journal (2015) 9, 2620-2629; doi:10.1038/ismej.2015.71; published online 19 May 2015
\end{abstract}

\section{Introduction}

Since the discovery of coral-hosted Symbiodinium, nutrition has been a major focus of coral symbiosis. Hand and Muscatine (1958) were among the first to demonstrate that Symbiodinium provide photosynthates to their hosts, thus settling debate over the functional significance of the intracellular algae. Since then, numerous studies have quantified the contribution of autotrophic and heterotrophic nutrition to the coral holobiont for a number of scleractinian species, even to sub-cellular scales

Correspondence: DM Baker, School of Biological Sciences, Kadoorie Biological Sciences Bldg 3 S-11, The University of Hong Kong, Pokfulam Rd, Hong Kong, PRC.

E-mail: dmbaker@hku.hk

${ }^{7}$ Current address: School of Natural Sciences, University of California, 5200 North Lake Rd, Merced, CA 95343, USA

Received 15 August 2014; revised 23 February 2015; accepted 1 April 2015; published online 19 May 2015
(Yellowlees et al., 2008; Houlbreque and FerrierPages, 2009; Pernice et al., 2014). We now know that Symbiodinium translocate carbon-based photosynthates in excess of host needs and nitrogen in the form of amino acids derived from host wastes and dissolved inorganic nitrogen from the water column. By coupling the coral polyp's opportunistic heterotrophy with Symbiodinium photosynthesis, the coral holobiont can thrive despite the severe nutrient limitation typical of tropical seas (Yellowlees et al., 2008; Houlbreque and Ferrier-Pages, 2009). However, holobiont performance and host benefits are poorly understood across different host-symbiont interactions and changing environmental conditions.

Our understanding of how coral-Symbiodinium interactions vary across diverse associations remains limited. Most studies have focused on several model coral species such as Stylophora pistillata, Pocillopora damicornis and members of the genus Acropora (Muller-Parker et al., 1994; Hoegh-Guldberg and 
Williamson, 1999; Grover et al., 2008). These species of photophilic corals all possess branching colony morphologies and small polyps and are thus too similar in their morphology and nutritional physiology to reveal the full breadth of coral-Symbiodinium functional diversity (Tremblay et al., 2012; Baker et al., 2013). Fewer nutritional studies have compared species within and between genera and even fewer in non-scleractinian anthozoans (Lasker, 1981; Lasker et al., 1983; Wilkerson and Muscatine, 1984; Ribes et al., 1998; Swanson and Hoegh-Guldberg, 1998). Such critical gaps limit our understanding of the functional, ecological and evolutionary significance of these symbioses.

The evolution of feeding structures occurs when species compete for limited nutritional resources (Grant and Grant, 2006). For corals, niche partitioning and competition likely drive the diversity of colony and polyp morphologies. Divergence towards the utilization of novel resources at the extremes of the heterotrophic - autotrophic continuum (that is, from plankton to dissolved inorganic nutrients) can reduce competitive interactions and drive speciation. Porter (1976) argued that variation in coral colony morphology, specifically the ratio of surface area to volume (SA:V), is central to understanding the nutritional strategies of scleractinian corals. Indeed, Porter predicted that autotrophy should be maximized in corals with high $\mathrm{SA}: \mathrm{V}$, with the specific idea that branching corals would exhibit higher photosynthesis/respiration $(\mathrm{P} / \mathrm{R})$ relative to massive (non-branching) species. Moreover, such a relationship would promote the evolution of 'layered' colony morphologies, which Porter identified as a convergent character in terrestrial plants. At a smaller scale, variation in polyp size is often posited as an indicator of heterotrophic capacity as larger polyps possess larger tentacles and gastrovascular cavities for prey capture and digestion. Conversely, small polyps have higher SA:V, which maximizes the light received by Symbiodinium, inhabiting the gastroderm at densities up to $10^{6}$ cells $\mathrm{cm}^{-2}$, and increases the surface area for nutrient uptake. Taken together, increasing or decreasing SA: $\mathrm{V}$ can occur at the colony and polyp scale. Here we test Porter's hypothesis by quantifying productivity (P/R and ${ }^{13} \mathrm{C}$ assimilation) and utilizing morphological, phylogenetic and ecological data on a diverse collection of Caribbean octocorals (gorgonians).

In the Caribbean Sea, gorgonians hosting Symbiodinium are especially diverse with at least 51 described species (Sánchez and Wirshing, 2005). Moreover, there is great variation in gorgonian morphology, with many reefs dominated by species with fan, plume or rod morphologies. Perhaps due to the morphological similarity to aposymbiotic deep water species, and the paucity of symbiotic species in the Indo-Pacific, there is a perception that symbiotic gorgonians gain most of their nutrition via passive suspension feeding (Leal et al., 2014). To date, the evidence to support this contention is limited. Indeed, symbiotic gorgonians differ in their ability to capture particles from the water column (Lasker, 1981). Although some species obtain sufficient carbon from particulate organic matter (Murdock, 1978; Coffroth, 1984), they still require nitrogen supplemented via Symbiodinium photosynthesis (Wainwright, 1967). Unlike Indo-Pacific soft corals (Fabricius and Klumpp, 1995) previous studies of Caribbean gorgonian productivity (as determined by oxygen metabolism) support a predominantly autotrophic nutrition in many species.

Here we expand on previous gorgonian productivity studies by using light and dark bottle incubations to estimate $\mathrm{P} / \mathrm{R}$ in combination with inorganic stable carbon isotope tracer additions to assess autotrophic productivity among 11 species of symbiotic gorgonians. We test the following null hypotheses: (1) all gorgonian symbioses are mutualistic where the host benefits from symbiont photosynthesis, (2) the mutualisms are invariant with respect to the amount of carbon translocated to the host and (3) the hostderived benefits of mutualism are unrelated to morphology, (that is, polyp size) of a species. Further, we examined the functional significance of varied productivity through a posteriori correlations between productivity measures and eco-physiology, including symbiont specificity and thermal bleaching resistance.

\section{Materials and methods}

\section{Field collections}

Five whole colonies of 11 gorgonian species were collected using SCUBA from reefs nearby the Smithsonian Tropical Research Institute's (STRI) field station in Bocas del Toro, Panama in September 2010. A total of three sites (depths of 3-8 m) were visited to obtain a diverse collection. The sea plume, Antillogorgia acerosa (AACE), and sea rods Pterogorgia anceps (PANC) and Eunicea succinea (ESUC) were collected from Boca del Drago $\left(9^{\circ} 25^{\prime} 21.52^{\prime \prime N}\right.$; $\left.82^{\circ} 19^{\prime} 35.78 " \mathrm{~W}\right)$. The sea rods Plexaura homomalla (PHOM), Plexaurella fusifera (PFUS), Plexaurella nutans (PNUT), Eunicea calyculata (ECAL), Eunicea flexuosa (EFLE), Eunicea mammosa (EMAM), sea plume Antillogorgia acerosa and sea fan Gorgonia ventalina (GVEN) were sampled from Crawl Cay Channel $\left(9^{\circ} 15^{\prime} 36.88^{\prime \prime N}\right.$; 82 $\left.{ }^{\circ} 6^{\prime} 32.78^{\prime \prime} \mathrm{W}\right)$. The sea rod Briareum asbestinum (BASB) was collected near South Carenaro Island $\left(9^{\circ} 20^{\prime} 36.19 " \mathrm{~N} ; 82^{\circ}\right.$ $\left.14^{\prime} 17.15^{\prime \prime W}\right)$. A subsample from each collected specimen was treated with dilute bleach to isolate sclerites, which were used to establish species identification following Bayer (1961). Calyx depth, the distance from the surface edge of the aperture (where the polyp emerges from the colony) to the base where the polyp is anchored within the colony, was used as a proxy for polyp size (Thibaudeau, 1983). The calyx is the anatomical space occupied by a single polyp, from which the polyp can extend into the environment. 
$P / R$ and ${ }^{13} C$ Incubation Experiment

Detailed experimental procedures are provided as Supplementary Online Material. In short, five colonies of each gorgonian species were fragmented into three clonal replicates, with one replicate preserved as an initial control. The remaining replicates were incubated in light and dark bottles for an assessment of net primary production and respiration, respectively, measured as change in dissolved oxygen concentration following Freeman et al. (2013). Seawater in each bottle was amended with $98 \%$ $\mathrm{NaH}^{13} \mathrm{CO}_{3}$, such that the resulting carbon stable isotope ratios $\left(\delta^{13} \mathrm{C}\right)$ could be used to trace photosynthetic assimilation by Symbiodinium and subsequent transfer to host cells (Baker et al., 2013; Freeman et al., 2013).

\section{Stable isotope analysis}

For details of sample preparation please refer to the Supplementary Online Methods. In brief, each dried gorgonian fragment was physically homogenized, and the host and symbiont fractions were separated using centrifugation of a suspended homogenate. The supernatant (host) and pellet (symbiont) were dispensed into silver capsules, acidified and dried before stable isotope analysis.

All samples were analyzed using a Thermo Delta V isotope ratio mass spectrometer coupled to a CarloErba NC2500 elemental analyzer via a Conflo III open-split interface at the Geophysical Laboratory. Analytical precision was determined by repeated analysis of an internal acetanilide standard ('acet 6'; $70 \% \mathrm{C})$. Standard precision during runs of initials and dark incubated enriched samples was $+/-0.2 \%$, and + - $-0.4 \%$ for the light incubated enriched samples.

Gorgonian phylogenetic signal and trait correlation Sequences of two mitochondrial protein-coding genes, ND2 and msh1, were obtained from GenBank to construct a phylogeny of the species sampled in this study ( McFadden et al., 2006; for details of tree construction see Supplementary Online Methods; Supplementary Table S2). Phylogenetic signal describes the degree to which more closely related organisms share more similar traits. We used the 'phylosignal' function of the $\mathrm{R}$ package Picante to test whether $\mathrm{P} / \mathrm{R}$ ratios, $\delta^{13} \mathrm{C}_{\text {host }}, \delta^{13} \mathrm{C}_{\text {svmbiont }}, \mathrm{C}: \mathrm{N}_{\text {host }}$, C: $\mathrm{N}_{\text {symbiont }}$ and calyx depth displayed significant phylogenetic signal given the host phylogeny (that is, whether more similar values were associated with more closely related gorgonians more often than expected by chance; refer to Supplementary Online Methods for further detail and references).

\section{A posteriori tests}

Following our experimental work, we obtained data from (1) GeoSymbio, a quality controlled database containing phylogenetic data on Symbiodinium isolated from diverse invertebrate hosts including gorgonians (Franklin et al., 2012), and (2) Prada et al. (2010), the most comprehensive report on gorgonian thermal bleaching (loss of Symbiodinium) from the 2005 mass coral bleaching event in Puerto Rico. These data were used to test the hypotheses that symbiont specificity and bleaching resistance are correlated with our productivity measurements (P/R, $\delta^{13} \mathrm{C}_{\text {host }}$ and $\delta^{13} \mathrm{C}_{\text {symbiont }}$ ).

\section{Results}

Gorgonian productivity

$\mathrm{P} / \mathrm{R}$ values varied significantly among the 11 species of Caribbean gorgonians measured (ANOVA; $F=5.26 ; P<0.0001$; Figure 1). All sea rods had average $P / R$ values $<1.5$, which is below a conservative threshold for autotrophy (Wilkinson, 1983). Five out of nine sea rod species had mean values below compensation $(\mathrm{P} / \mathrm{R}=1.0)$. In contrast, a sea fan (G. ventalina) and sea plume (A. acerosa) were both net autotrophs with $\mathrm{P} / \mathrm{R} \sim 1.8$ with some replicate fragments exceeding 2.5. $\mathrm{P} / \mathrm{R}$ was strongly correlated with autotrophy ( $\mathrm{NPP}_{\text {light }} ; R^{2}=0.84, P<0.0001, n=11$ ), not respiration $\left(\mathrm{NPP}_{\text {dark }} ; R^{2}=0.16, P=0.22, n=11\right.$; Supplementary Table S1). However, the highest dark bottle respiration was observed in A. acerosa (mean NPP $=-2.03 \mathrm{mg} \mathrm{O}_{2} \mathrm{l}^{-1} \mathrm{~g}^{-1} \mathrm{~h}^{1}$ ) and generally declined with increasing colony volumes with $P$. nutans and $P$. fusifera having the lowest observed respiration (Supplementary Table S1).

$\delta^{13} \mathrm{C}$ - natural abundance and tracer experiment

Natural abundance $\delta^{13} \mathrm{C}$ ranged from -20.4 to $-14.9 \%$ with a mean of $-17.3 \%$. There was a strong correlation between the mean natural abundance $\delta^{13} \mathrm{C}_{\text {host }}$ and $\delta^{13} \mathrm{C}_{\text {symbiont }}$ inclusive of all species $\left(R^{2}=0.67 ; \quad P=0.002 ; \quad\right.$ Supplementary Figure S2). $\delta^{13} \mathrm{C}$ values increased to a mean of $-6.9 \%$ and $261 \%$ in the dark and light incubations, respectively (ANOVA; $F=252, P<0.001 ;$ Figure 2). In most species, ${ }^{13} \mathrm{C}$ enrichments in the light treatment were similar between the host and symbiont fractions (Figure 2a), but the light bottle $\delta^{13} \mathrm{C}_{\text {symbiont }}$ was a better predictor of holobiont $\mathrm{P} / \mathrm{R}$ than the corresponding $\delta^{13} \mathrm{C}_{\text {host }}\left(R^{2}=0.84\right.$ vs 0.67 , respectively; Supplementary Figure S2). Fragments incubated in the dark took up less ${ }^{13} \mathrm{C}$ owing to a lack of photosynthesis (Figure 2a). Yet, significant ${ }^{13} \mathrm{C}$ assimilation was observed in the host fractions of many species (Figure 2b). The $\delta^{13} \mathrm{C}_{\text {host }}$ of $P$. anceps, $A$. acerosa and $B$. asbestinum incubated in the dark were all $<10 \%$, greater than initial values, but much lower than the $\delta^{13} \mathrm{C}_{\text {host }}$ values from the light bottle treatment. In fact, all host fractions from the dark treatment were, on average, $+11.8 \%$ enriched relative to their Symbiodinium. 


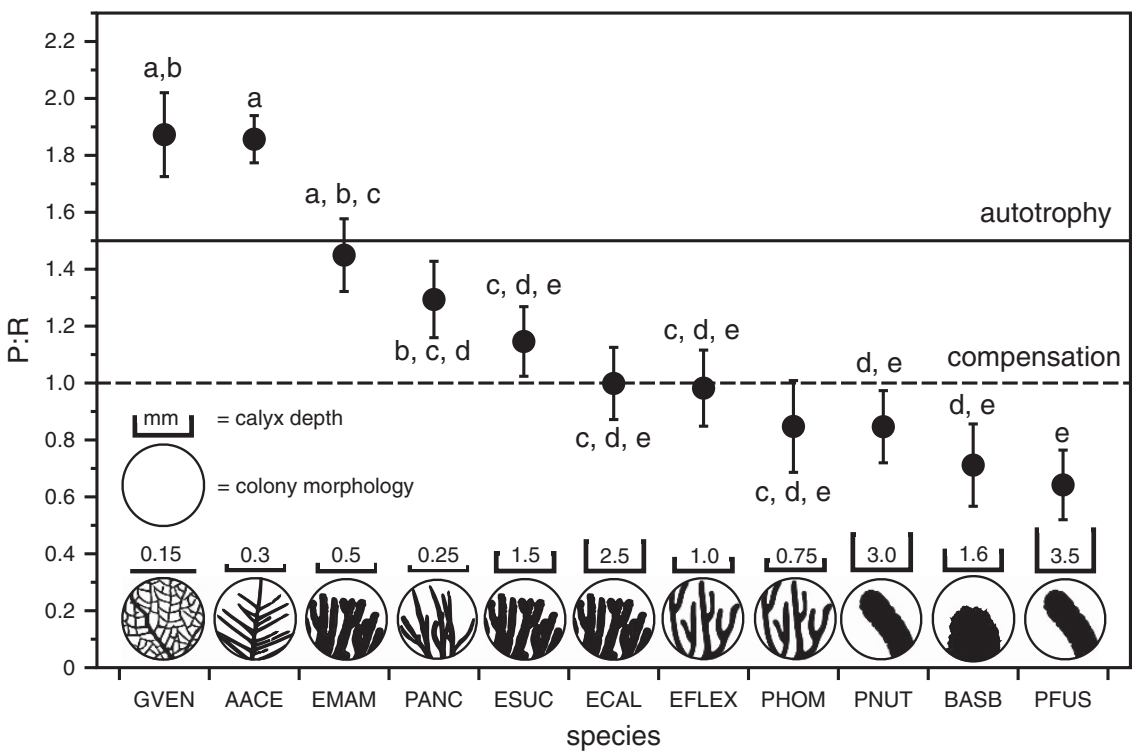

Figure 1 Least-squared mean P/R values from 11 species of symbiotic Caribbean gorgonians ordered from highest to lowest values. Dashed line defines compensation (where $\mathrm{P} / \mathrm{R}=1.0$ ), and the solid line a conservative threshold for autotrophy $(\mathrm{P} / \mathrm{R}>1.5 ;(\mathrm{Wilkinson}$, 1983)). $\mathrm{P} / \mathrm{R}$ was calculated from at least four pairs (one light, one dark treatment) of similarly sized clonal fragments from independent colonies $(n \geq 4)$. Error bars represent s.e.m. and letters indicate significant differences between groups as determined by Tukey's post hoc test $(P<0.05)$. For species abbreviations refer to the Methods section. Colony morphology (not to scale) and calyx depth (to scale in mm) are shown for reference.

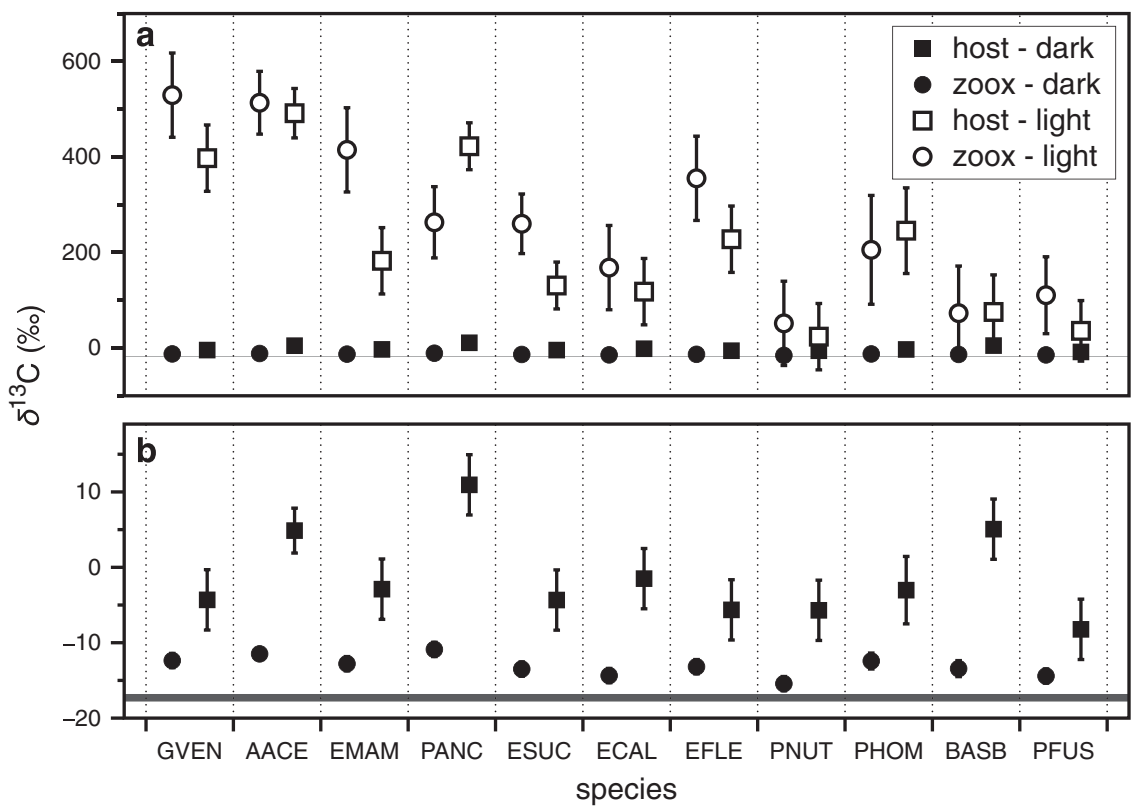

Figure 2 (a) Mean $\delta^{13} \mathrm{C}$ of 11 species of gorgonians and their Symbiodinium after a $\sim 7.5 \mathrm{~h}$ incubation in dark bottles (filled shapes) or light bottles (open shapes). (b) The same data for host and symbiont fractions incubated in darkness and with enlarged scale to illustrate differences. Gray horizontal lines indicate the upper and lower $95 \%$ confidence intervals of the mean natural abundance $\delta^{13} \mathrm{C}$ values for all species (initial samples). Species abbreviations are listed in the Methods section and are presented in order of decreasing P/R. Error bars represent the $95 \%$ confidence interval of the mean.

\section{Phylogenetic signals in metabolic traits}

The phylogeny of Octocorallia used in this study is a subset of the phylogeny reported by McFadden et al. (2006) based on ND2 and msh1 sequences. We found strong support for clades representing the genera Plexaurella and Eunicea, as well as a clade containing Antillogorgia and Gorgonia (Figure 3). Four traits displayed significant phylogenetic signal, including the enriched $\delta^{13} \mathrm{C}_{\text {host }}$ and $\delta^{13} \mathrm{C}_{\text {symbiont }}$ values, P:R and calyx depth (Supplementary Table S3). The two most significant traits, $\delta^{13} \mathrm{C}_{\text {host }}$ and calyx depth, demonstrated a significant negative correlation under both models of trait covariance (BM: $r=$ -0.450, $\quad P=0.044$; $\quad$ OU: $\quad r=-0.83, \quad P<0.001$; Figure 3). Calyx depth was negatively correlated with $\mathrm{P} / \mathrm{R}\left(R^{2}=0.65 ; P=0.005\right.$; Figure 4$)$. 


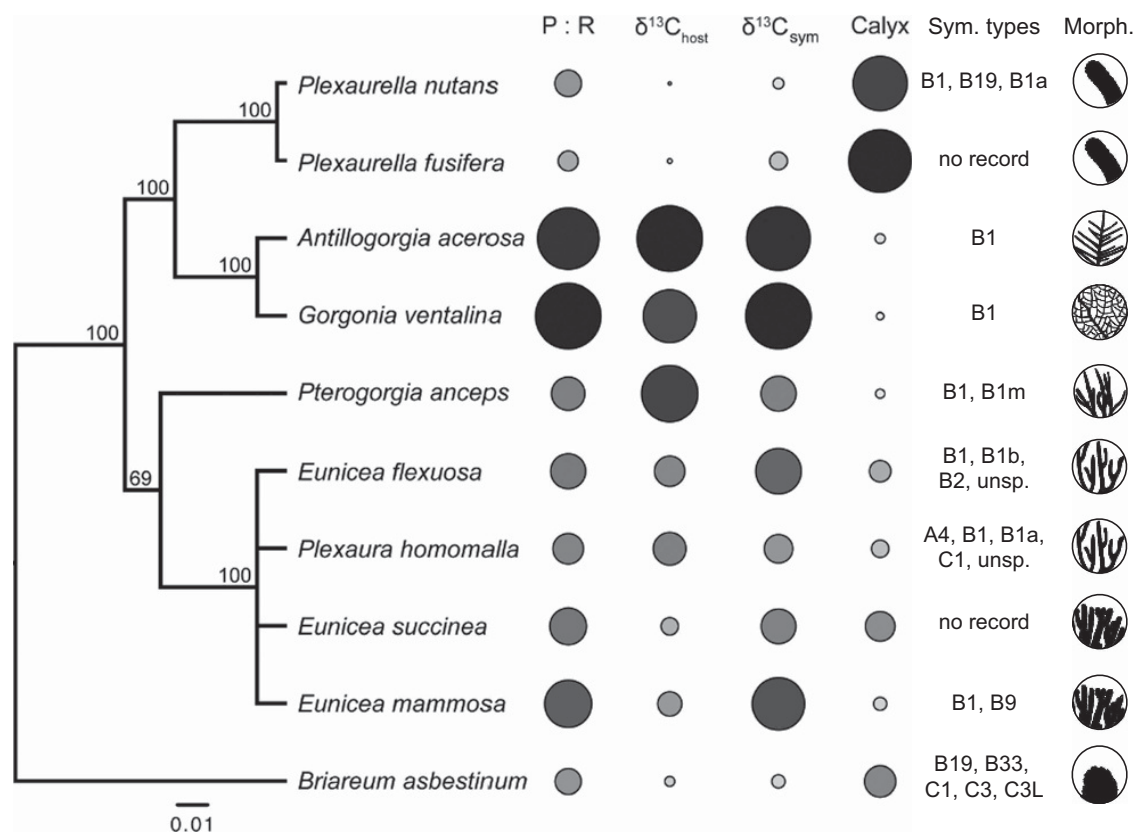

Figure 3 Phylogeny of Octocorallia used in this study, derived from sequences reported by McFadden et al. (2006). The scale bar indicates number of substitutions per site, while numbers at nodes indicate percentage Bayesian posterior probabilities. To the right of the phylogeny, four columns of circles represent values of photosynthesis to respiration ratios $(\mathrm{P} / \mathrm{R}), \delta^{13} \mathrm{C}_{\mathrm{host}}, \delta^{13} \mathrm{C}_{\text {symbiont }}$ and calyx depth (used as a proxy for polyp size). The size and shading of each circle are proportional to the values reported in Supplementary Table S1. In addition, the known Symbiodinium associations are shown as well as the colony morphology (not to scale). 'unsp.' Indicates a unique Symbiodinium genotype with no clade designation.

A posteriori tests - specificity and bleaching

Using quality-controlled records obtained from the GeoSymbio database (Franklin et al., 2012), we found that the gorgonian species studied herein host one to five unique Symbiodinium types throughout the Caribbean Sea (Figure 3; Supplementary Table S1). G. ventalina and Antillogorgia have only been found in association with clade B1 suggesting a highly specific partnership. In contrast, records from all sea rods suggest more generalist associations with low specificity for a given Symbiodinium type, ranging from two reported species in Pterogorgia and E. mammosa, up to five in B. asbestinum including B19, B33, C1, C3 and C3L (Figure 3; Supplementary Table S1). Taken together, $85 \%$ of the variation in holobiont $\mathrm{P} / \mathrm{R}$ was explained by the number of known Symbiodinium genotypes associated with each host species (Figure 5).

Using data extracted from Prada et al. (2010) for 8 of 11 of our studied species, we found that P/R explained $33 \%$ of the variation in bleaching prevalence (Figure 6). However, when $P$. anceps was excluded from this analysis, the explained variation improved to $96 \%$. Excluding $P$. anceps may be appropriate as this species often exhibits white polyps that could be mistaken for a bleached condition (pers. obs.). The logistic fit illustrates that species with $\mathrm{P} / \mathrm{R}$ values $<1.1$ are highly susceptible to bleaching. Conversely, corals bearing small polyps with high $\mathrm{P} / \mathrm{R}$ appear to resist bleaching.

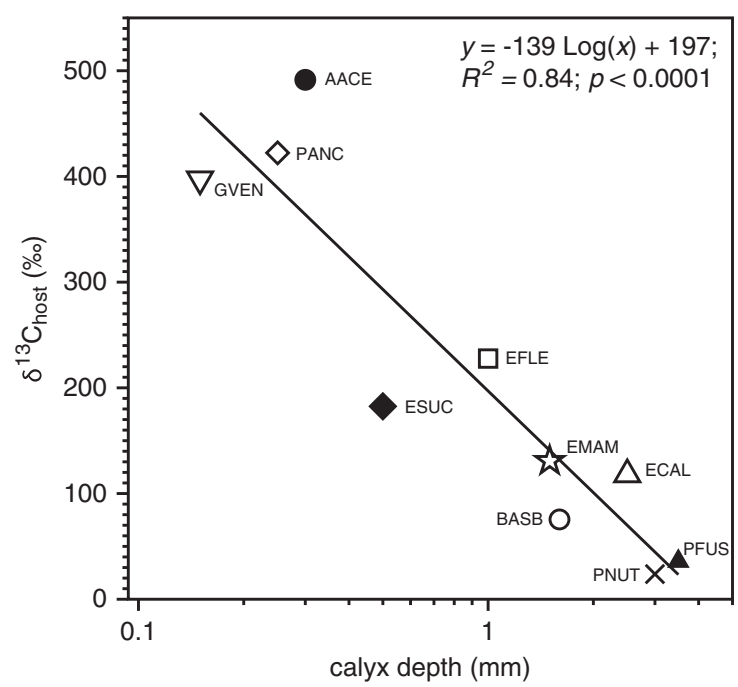

Figure 4 Enriched $\delta^{13} \mathrm{C}_{\text {host }}$ values as a function of calyx depth (used as a proxy for polyp size) for eight species of gorgonians (data from Thibaudeau, 1983). Solid line represents a significant relationship between the variables. For species abbreviations refer to the Methods section.

\section{Discussion}

Gorgonian productivity varies with morphology

Our data show that the sea fan $G$. ventalina and plume $A$. acerosa produced $>2$ times their required carbon demand with $\mathrm{P} / \mathrm{R}$ values similar to many scleractinian corals. As such, we conclude that these species are net autotrophs, acquiring the majority of 


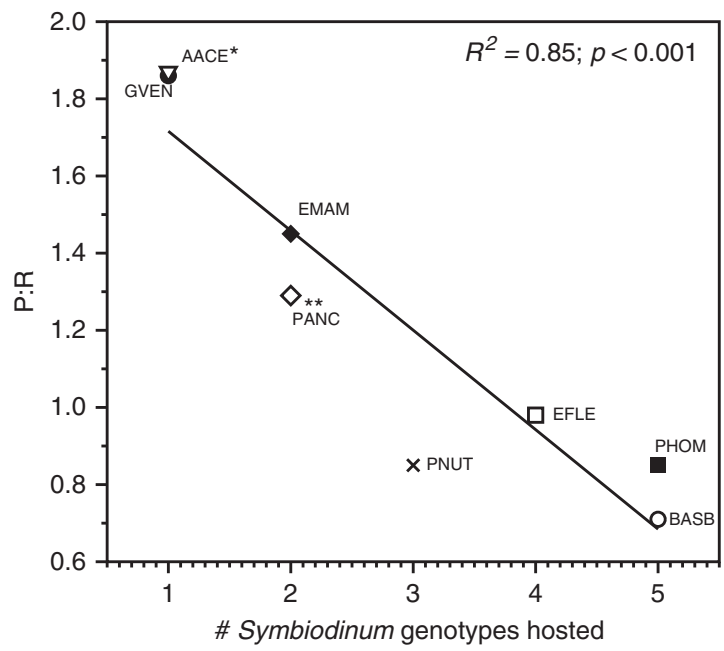

Figure 5 Observed Symbiodinium genotypes (based on ITS2) obtained from the GeoSymbio database, representing eight gorgonian species analyzed in this study. All $n \geqq 3$. *Indicates $\mathrm{P} / \mathrm{R}$ data for $A$. acerosa, using symbiont records for $A$. elisabethae and $A$. bipinnata as there are no records for $A$. acerosa. **Indicates $\mathrm{P} / \mathrm{R}$ for $P$. anceps and symbiont records for all Pterogorgia as there was only one record for P. anceps. For species abbreviations refer to the methods section.

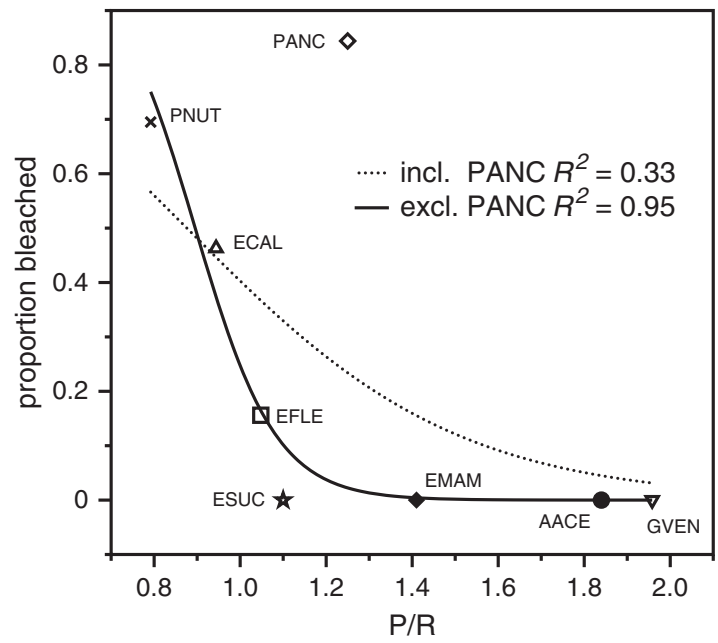

Figure 6 Observed prevalence of bleaching of eight gorgonian species during the 2005 mass bleaching in Puerto Rico (data from Prada et al. (2010)) as a function of gorgonian P/R. The dashed line represents a logistic function fit to all data, whereas the solid line represents a logistic function fit to all data excluding $P$. anceps. For species abbreviations refer to the Methods section.

their nutrition from Symbiodinium photosynthesis. This contrasts with all sea rod species that had $\mathrm{P} / \mathrm{R}<1.5$ and all Indo-Pacific alcyonaceans that have negative P/R values (Fabricius \& Klumpp, 1995). Species with fan or plume morphologies and small polyps might maximize light exposure and optimize symbiont densities and nutrient exchange via increased SA:V. Similar observations have been made for Indo-Pacific sponges, which are more productive than their Caribbean counterparts with highly autotrophic species exhibiting flattened morphologies (encrusting, fan, dish) that are convergent traits in corals (Wilkinson, 1983).

In one of the first studies of gorgonian metabolism, Cary (1918) measured respiration in 12 Caribbean species and found that the highest rates of respiration were observed in species with the highest SA:V (that is, Gorgonia, Antillogorgia and Pterogorgia). Thus, we hypothesize that some gorgonian species are more metabolically active than others, as positive NPP is maintained by high rates of photosynthesis, despite substantial respiration. Therefore, observed variations in $\mathrm{P} / \mathrm{R}$ are due to overall metabolic rates rather than variation in either photosynthesis or respiration alone. Like Kanwisher and Wainwright (1967), we found a wide range of $P / R$ values for Caribbean gorgonians, which followed a general pattern of increasing $\mathrm{P} / \mathrm{R}$ with $\mathrm{SA}: \mathrm{V}$. We stress, however, that comparisons among disparate studies should be interpreted with caution, as the method of oxygen measurement and environmental conditions can vary considerably between studies. Nevertheless, it is interesting that Caribbean gorgonians have a similar range of $\mathrm{P} / \mathrm{R}$ values to some Indo-Pacific hard corals, although our data are lower than previous reports (Roffman, 1968).

Porter (1976) put forth several hypotheses about functional morphology of corals that are consistent with our observations on gorgonians. First, the parallels drawn between tree morphology (sensu Horn (1971)) are particularly striking as the most autotrophic species ( $G$. ventalina and $A$. acerosa) have morphologies similar to the broad leaves and needles of trees, respectively, which are plant adaptations for enhancing photosynthesis while moderating transpiration. In sea plumes, alternating branches reduce selfshading and permit light transmission to lower portions of the colony. Sea fans have a reticulate and planar morphology with high surface area. Over time, sea fans twist their axial skeleton to a position perpendicular to water flow (Wainwright and Dillon, 1969). It has been suggested that fan morphologies aid in particle capture (Leversee, 1976). While this may be true for deep-water sea fan species, for $G$. ventalina found in high energy, shallow environments this orientation facilitates the oscillation of the colony from full illumination to shade in synchrony with the wave period. This also permits the photosynthetic centers to recover from light saturation and maximize photosynthetic efficiency over the course of the day. Colonies uprooted or twisted from this position often show heavy melanization (purpling) in response to excessive light stress to one face of the fan, whereas adjacent colonies with normal skeletal development/positioning exhibit normal coloration (pers. obs.).

Alternatively, the cylindrical or bladed, digitate and branching forms common to the various genera of sea rods may be more suited to capturing plankton and particulate matter, especially those with larger polyp sizes. Thick, rounded colony branches may function to generate eddies on the side of the branch opposite an oncoming current. Such eddies can 
cause plankton and particulate matter to linger, affording a polyp more opportunity for capture (Leversee, 1976). There is limited evidence to suggest that larger polyps facilitate prey capture. Lasker et al. (1983) found that two sea rods, Plexaura homomalla and P. nina, found on the shallow and deep forereef, respectively, differed in their prey capture abilities. With twice the polyp size, $P$. nina was able to capture more particles. It is interesting that the difference in polyp size and distribution of these congeners occurs over a depth gradient, which suggests that larger polyps are advantageous in deeper habitats where light is limiting.

\section{$\delta^{13} \mathrm{C}$ as an indicator of trophic position}

The natural abundance isotope values of gorgonians are useful indicators of both diet and localized variation in the base of the marine food web. Our data show a wide range in $\delta^{13} \mathrm{C}$ values (nearly 3.5\%) and a strong correlation between the $\delta^{13} \mathrm{C}_{\text {host }}$ and $\delta^{13} \mathrm{C}_{\text {symbiont }}$, indicating either a common carbon source or tight cycling of carbon between host and symbiont (Supplementary Figure S1). Interestingly, there is little support for utilization of POM in the gorgonian diet. POM from Bocas del Toro had a $\delta^{13} \mathrm{C}$ of $-23 \%$ (Freeman and Thacker, 2011), which is a reflection of a large terrestrial contribution to Almirante Bay (Aronson et al., 2014). The average $\delta^{13} \mathrm{C}$ of gorgonians $(-17.4 \%$ ) greatly exceeded the $1 \%$ trophic enrichment expected between a consumer and its diet. Instead, these values may be enriched via photosynthetic assimilation of dissolved inorganic carbon (DIC; $\delta^{13} \mathrm{C}_{\mathrm{HCO}-} \approx 0 \%$ ). As such, gorgonian $\delta^{13} \mathrm{C}$ is a weighted average of DIC and POM contributions to the auto- and heterotrophic diet, respectively. Ultimately, we limit our interpretation of these data as different environmental sources and conditions among the three collection sites preclude a detailed view of the trophic position of gorgonians based on natural abundance $\delta^{13} \mathrm{C}$. In this regard, the ${ }^{13} \mathrm{C}$ tracer study was effective in highlighting the relative utilization of DIC via photosynthesis among these species.

\section{${ }^{13} \mathrm{C}$ Tracer reveals the benefits of symbiosis}

We have shown that Caribbean gorgonians receive important carbon resources from their associated Symbiodinium, which drives significant variation in productivity among species. The autotrophic assimilation of $\mathrm{H}^{13} \mathrm{CO}_{\overline{3}}$ in the light bottles was evident in $\delta^{13} \mathrm{C}_{\text {host }}$ and $\delta^{13} \mathrm{C}_{\text {symbiont }}$, suggesting a tightly coupled and rapid translocation of ${ }^{13} \mathrm{C}$-labeled carbohydrates and other biomolecules during the brief incubation (Figure 2a). The higher $R^{2}$ observed between $\delta^{13} \mathrm{C}_{\text {svmbiont }}$ and P/R (Supplementary Figure S2) may indicate superior precision of direct quantification of ${ }^{13} \mathrm{C}$ assimilation by the holobiont versus the relatively indirect calculation of productivity based on oxygen metabolism from clonal fragments within separate bottles.
The relative enrichment of $\delta^{13} \mathrm{C}_{\text {host }}$ attained versus $\delta^{13} \mathrm{C}_{\text {symbiont }}$ reflects the variation in photosynthate translocated from source (symbiont) to sink (host). Eunicea mammosa and E. succinea had the lowest relative $\delta^{13} C_{\text {host }}$ enrichment. Such low $\delta^{13} \mathrm{C}_{\text {host }}$ could result from several factors including (1) higher symbiont metabolism relative to the host, (2) limited translocation of photosynthate due to lower host SA:V, (3) differential host and symbiont biomass, and similarly, (4) a relatively larger structural pool of carbon in the host fraction, which 'dilutes' any translocated ${ }^{13} \mathrm{C}$. However, if these factors were important in determining the final ${ }^{13} \mathrm{C}$ assimilation in the host, then similar patterns would have been observed in the Plexaurella, which have the largest polyps, lowest SA:V, and thickest host tissues among the species studied. Given that this is not the case, an alternative explanation is that Symbiodinium within $E$. mammosa and E. succinea are less mutualistic, retaining more of their photosynthates for their own metabolism. Such observations of cheating have been reported for a variety of microbial symbioses (Bronstein, 2001; Porter and Simms, 2014). This contrasts with species like $P$. anceps that achieved higher $\delta^{13} \mathrm{C}_{\text {host }}$ than $\delta^{13} \mathrm{C}_{\text {symbiont }}$, suggesting a larger carbon subsidy to the host and therefore a more mutualistic relationship. Interestingly, $P$. anceps had the highest natural abundance $\delta^{13} \mathrm{C}$ values reported in this study (Supplementary Figure S1), suggesting a large contribution of autotrophically derived DIC.

Although ${ }^{13} \mathrm{C}$ assimilation was not observed in any dark-incubated Symbiodinium, it was curiously evident in all host fractions (Figure 2b). The former is easily explained by a lack of light to drive photosynthesis. In the dark, host enrichment in the absence of symbiont photosynthesis must be proceeding by anaplerotic pathways, such as fatty-acid synthesis and oxidation (DeNiro and Epstein, 1977) or by associated microbiota. Microbes other than Symbiodinium could contribute to ${ }^{13} \mathrm{C}$ enrichment through similar biosynthetic pathways, as their signal is inseparable from the host fraction. Quantifying the contributions of associated chemoautotrophic microbes such as cyanobacteria and archaea to holobiont productivity and biogeochemical cycling warrants further study. However, the large differences in observed $\delta^{13} \mathrm{C}_{\text {host }}$ enrichments ( $>3$ to 30 -fold) between the light and dark treatment suggest that associated microbe ${ }^{13} \mathrm{C}$ assimilation was minimal relative to Symbiodinium.

We found that not all hosts benefit from Symbiodinium autotrophy. Interestingly, there was no effect of light on Plexaurella or Briareum $\delta^{13} \mathrm{C}_{\text {host }}$ and $\delta^{13} \mathrm{C}_{\text {symbiont }}$ (except $P$. fusifera $\delta^{13} \mathrm{C}_{\text {symbiont }}$ ). Given that marginal enrichments of the $\delta^{13} \mathrm{C}_{\text {host }}$ can be attributed to light-independent assimilation (Figure 2b), we conclude that Symbiodinium hosted by $P$. fusifera, $P$. nutans and $B$. asbestinum did not translocate carbon to their hosts. Indeed, the three species were among the lowest with regards to $\mathrm{P} / \mathrm{R}$ with mean values <1.0. As such, the interaction between 
Symbiodinium and large polyp gorgonian hosts may be more commensal than mutualistic, at least as it pertains to carbon metabolism. However, even though observed ${ }^{13} \mathrm{C}$ assimilation was low in many sea rod species, the role of Symbiodinium may still be critically important for obtaining growth-limiting resources such as nitrogen (Ribes et al., 1998).

\section{Symbiont identity and gorgonian productivity}

Caribbean gorgonians generally associate with members of Symbiodinium clade B (Baker et al., 2013), which is common on Caribbean reefs (Pettay and Lajeunesse, 2007). Certain sub-clade types such as B1 are especially common, leading to the conclusion that this is a generalist symbiont associated with many hosts (LaJeunesse, 2002). However, Finney et al. (2010) revealed that this clade contains substantial genetic variation with evidence of highly specialized symbionts among various host species. Such specialization could occur during divergence among host species, such has been reported for depth partitioned lineages of E. flexuosa and co-evolution of their Symbiodinium (Prada et al., 2014). Our comparison of known Symbiodinium types found evidence of symbiont specificity increasing with holobiont productivity (Figure 5). Generalist hosts had lower productivities in comparison with specialists, which exhibited a higher degree of autotrophy. The mechanism underlying the enhanced metabolism afforded by specialized symbioses, including the functional significance of octocoral-associated Symbiodinium clade B warrants further investigation (Baker et al., 2013; Pernice et al., 2014).

\section{Bleaching and gorgonian productivity}

During the 2005 mass coral bleaching in Puerto Rico only 8 out of 24 gorgonian species showed visible signs of bleaching among four monitored reefs. Remarkably, with the exception of Muricea, no species suffered subsequent mortality (Prada et al., 2010). However, the corals that readily bleached were species with larger polyps and, as we have reported here, lower rates of productivity (Figure 6). Considering the observed specificity among highly productive species, we predict that such resistance to bleaching is reflective of the obligate nature of these symbioses. Essentially, bleaching may be disadvantageous to an obligate host if nutrition is derived exclusively from symbionts. This explains the difficulty in experimentally bleaching $G$. ventalina by elevated temperatures (Kirk et al., 2005), prolonged incubation in darkness, or with the herbicide DCMU (Baker, unpublished data). Conversely, in more facultative interactions hosts are more likely to expel symbionts when the costs (such as nutrient retention, increased respiration, or oxidative damage) outweigh the nutritional benefits, especially if the host can alternatively acquire resources easily by heterotrophy. Further work is needed to test these hypotheses among other Symbiodinium - cnidarian symbioses.

Gorgonian metabolism and the evolution of symbiosis Across the diversity of gorgonians, there is a strong negative correlation between traits associated with heterotrophy and autotrophy, reflecting strong evolutionary and ecological trade-offs between their divergent life-history strategies. Indeed, we found a link between productivity and the evolutionary trajectories of host species, which may be driven by habitat specialization and niche partitioning within highly competitive coral reef environments. The strong negative phylogenetic correlation observed between polyp size and carbon translocated from symbiont to host suggests that there is an evolutionary trade-off between heterotrophic and autotrophic modes of nutrition. Species like $G$. ventalina and $A$. acerosa possess very small polyps compared with some sea rods like Eunicea and Plexaurella, whose polyps are up to an order of magnitude larger (Thibaudeau 1983). As a consequence, the density and surface area of each polyp, relative to the overall colony size is much higher for the sea fans and plumes than the sea rods we studied. Thus, selection may favor the reduction of feeding structure $\mathrm{SA}: \mathrm{V}$ if it increases the amount of light exposure to productive symbionts in the host polyp tissues (Kanwisher and Wainwright, 1967; Wainwright and Dillon, 1969). The converse appears to be true for most sea rods, where larger and fewer polyps and the thick, rod shaped colony morphology would serve to limit productivity per unit of biomass but ensure that feeding structures are ready for heterotrophy during changing environmental conditions.

Over deep time, gorgonian diversification in the Caribbean was less inhibited by competitive interactions with scleractinians than in the Pacific. With more niche space available, any species that acquired mutualistic Symbiodinium could boost its ability to compete for space. In doing so, selection may have favored more obligate associations with Symbiodinium with more efficient metabolisms leading to greater productivity and growth (and thus, competitive abilities) in oligotrophic environments. Such divergence is obvious within the Gorgoniidae, which display a stark contrast between clades formed by Gorgonia and Antillogorgia versus the Plexaurella (Figure 3). These two host species are apparent specialists with Symbiodinium clade B1 as reported by substantial sampling efforts throughout the Caribbean Sea (Andras et al., 2011). Moreover, this specialization may have accelerated the selection for reduced polyp size and colony morphologies to enhance light capture (Figure 3). A shared trajectory toward symbiont specificity and autotrophy is further supported by the observation that Gorgonia and Antillogorgia are capable of hybridization (McFadden et al., 2010). In contrast, we have shown that Plexaurella do not benefit substantially 
from Symbiodinium and hosts are less specific in their Symbiodinium associations (Figures 2 and 3). This may reflect a divergence from the Gorgonia/ Antillogorgia sister group to avoid competition for space in high light environments.

While we have examined the benefits of the gorgonian-Symbiodinium symbiosis, further work must address the potential costs of these interactions. For example, with changing environmental conditions (higher SST, eutrophication, acidification), do the costs of symbiosis outweigh the benefits? We hypothesize that host energetics and related physiological mechanisms (such as immunity to pathogens) could be a key indicator of the cost of symbiosis. For example, productive and obligate hosts like $G$. ventalina were nearly extirpated from the Caribbean by disease (Bruno et al., 2011), whereas other gorgonian species were not as impacted. The severity of this disease has been attributed to eutrophication (Baker et al., 2007). Thus, the cost of symbiosis, including the pairing of auto- and heterotrophic metabolisms could become an Achilles' heel when the environment changes.

For other species, there are benefits to being flexible in a symbiotic relationship particularly during periods of environmental change. The relatively heterotrophic sea rods (that is, Plexauridae including Eunicea) are Caribbean endemics undergoing rapid speciation. In our study, this group displayed variable metabolic performance (Figure 3). Indeed, Eunicea is the most diverse anthozoan genus with 16 described species (Sánchez et al., 2003). They are phenotypically plastic and can adapt to different habitat types along a reefscape, which contributes to their high rate of speciation (Prada and Hellberg, 2013; Prada et al., 2014). In the process of speciation, the association with Symbiodinium may weaken from an obligate mutualism to a commensal or parasitic relationship. In the Anthropocene, selection may favor facultative symbioses as the coastal oceans become more eutrophic and nitrogen limitation is alleviated (Baker et al., 2010). We predict that generalist and facultative hosts may be more resilient in the face of environmental stressors that specifically impact symbionts (reduced light) and capitalize on the increasing abundance of heterotrophic resources in the form of plankton and particulate organic matter to meet their nutritional needs.

\section{Conflict of Interest}

The authors declare no conflict of interest.

\section{Acknowledgements}

We thank Gondola, G Jacome, and the staff of the Smithsonian Tropical Research Institute's field station at Bocas del Toro for assistance with field and lab logistics. A Frederick assisted with sample preparation. R Bowden provided support for isotope analyses at the Geophysical Laboratory. Two anonymous reviewers provided constructive feedback. Financial support was provided by the Smithsonian Marine Science Network Fellowship to DMB, the Keck Foundation to MLF, and the U.S. National Science Foundation under grants 0829986 and 1208340 to RWT.

\section{References}

Andras JP, Kirk NL, Harvell CD (2011). Range-wide population genetic structure of Symbiodinium associated with the Caribbean Sea fan coral, Gorgonia ventalina. Mol Ecol 20: 2525-2542.

Aronson RB, Hilbun NL, Bianchi TS, Filley TR, McKee BA (2014). Land use, water quality, and the history of coral assemblages at Bocas del Toro, Panamá. Mar Ecol Prog Ser 504: 159-170.

Baker DM, Andras JP, Jordán-Garza A, Fogel ML (2013). Nitrate competition in a coral symbiosis varies with temperature among Symbiodinium clades. ISME J 7: 1248-1251.

Baker DM, MacAvoy SA, Kim K (2007). Relationship between water quality, d15N, and aspergillosis of Caribbean sea fan corals. Mar Ecol Prog Ser 343: 123-130.

Baker DM, Webster KL, Kim K (2010). Caribbean octocorals record changing carbon and nitrogen sources from 1862 to 2005. Glob Change Biol 16: 2701-2710.

Baker DM, Weigt L, Fogel M, Knowlton N (2013). Ancient DNA from coral-hosted Symbiodinium reveal a static mutualism over the last 172 years. PLoS One 8: e55057.

Bayer FM (1961). The shallow-water Octocorallia of the West Indian region; A Manual for Marine Biologists. Martinus Nijhoff: The Hague.

Bronstein JL. (2001). The costs of mutualism. Am Zool 41: 825-839.

Bruno JF, Ellner SP, Vu I, Kim K, Harvell CD (2011). Impacts of aspergillosis on sea fan coral demography: modeling a moving target. Ecol Monogr 81: 123-139.

Cary L (1918). A study of respiration in alcyonaria. Papers from the Department of Marine Biology 252: 187-191.

Coffroth M (1984). Ingestion and incorporation of coral mucus aggregates by a gorgonian soft coral. Mar Ecol Prog Ser 17: 193-199.

DeNiro M, Epstein S (1977). Mechanism of carbon isotope fractionation associated with lipid synthesis. Science 197: 261.

Fabricius K, Klumpp D (1995). Widespread mixotrophy in reef-inhabiting soft corals: the influence of depth, and colony expansion and contraction on photosynthesis. Mar Ecol Prog Ser 125: 195-204.

Finney JC, Pettay DT, Sampayo EM, Warner ME, Oxenford HA, LaJeunesse TC (2010). The relative significance of host-habitat, depth, and geography on the ecology, endemism, and speciation of coral endosymbionts in the genus Symbiodinium. Microb Ecol 60: 250-263.

Franklin EC, Stat M, Pochon X, Putnam HM, Gates RD (2012). GeoSymbio: a hybrid, cloud-based web application of global geospatial bioinformatics and ecoinformatics for Symbiodinium-host symbioses. Mol Ecol Res 12: 369-373.

Freeman CJ, Thacker RW (2011). Complex interactions between marine sponges and their symbiotic microbial communities. Limnol Oceangr 56: 1577-1586. 
Freeman CJ, Thacker RW, Baker DM, Fogel ML (2013). Quality or quantity: is nutrient transfer driven more by symbiont identity and productivity than by symbiont abundance? ISME J 7: 1116-1125.

Grant PR, Grant BR (2006). Evolution of character displacement in Darwin's Finches. Science 313: 224-226.

Grover R, Maguer J-F, Allemand D, Ferrier-Pages C (2008). Uptake of dissolved free amino acids by the scleractinian coral Stylophora pistillata. J Exp Biol 211: 860-865.

Hand C, Muscatine L (1958). Direct evidence for the transfer of materials from symbiotic algae to the tissues of a coelenterate. Proc Natl Acad Sci USA 44: 1259-1263.

Hoegh-Guldberg O, Williamson J (1999). Availability of two forms of dissolved nitrogen to the coral Pocillopora damicornis and its symbiotic zooxanthellae. Mar Biol 133: 561-570.

Horn HS (1971). The Adaptive Geometry of Trees. Princeton University Press.

Houlbreque F, Ferrier-Pages C (2009). Heterotrophy in Tropical Scleractinian Corals. Biol Rev 84: 1-17.

Kanwisher J, Wainwright S (1967). Oxygen balance in some reef corals. Biol Bull 133: 378.

Kirk N, Ward J, Coffroth M (2005). Stable Symbiodinium composition in the sea fan Gorgonia ventalina during temperature and disease stress. Biol Bull 209: 227-234.

LaJeunesse T (2002). Diversity and community structure of symbiotic dinoflagellates from Caribbean coral reefs. Mar Biol 141: 387-400.

Lasker $H$ (1981). A comparison of the particulate feeding abilities of three species of gorgonian soft coral. Mar Ecol Prog Ser 5: 61-67.

Lasker H, Gottfried M, Coffroth M (1983). Effects of depth on the feeding capabilities of two octocorals. Mar Biol 73: 73-78.

Leal MC, Berger SA, Ferrier-Pagès C, Calado R, Brandes J, Frischer ME et al. (2014). Temporal changes in the trophic ecology of the asymbiotic gorgonian Leptogorgia virgulata. Mar Biol 161: 2191-2197.

Leversee G (1976). Flow and feeding in fan-shaped colonies of the gorgonian coral Leptogorgia. Biol Bull 151: 344-356.

McFadden C, Sanchez J, France SC (2010). Molecular phylogenetic insights into the evolution of Octocorallia: a review. Int Comp Biol 50: 389-410.

McFadden CS, France SC, Sánchez JA, Alderslade P (2006). A molecular phylogenetic analysis of the Octocorallia (Cnidaria: Anthozoa) based on mitochondrial protein-coding sequences. Mol Phylogenet Evol 41: 513-527.

Muller-Parker G, Cook C, D'Elia C (1994). Elemental composition of the coral Pocillopora damicornis exposed to elevated seawater ammonium. Pac Sci 48: 234-246.

Murdock GR (1978). Circulation and digestion of food in the gastrovascular system of gorgonian octocorals (Cnidaria; Anthozoa). Bull Mar Sci 28: 363-370.

Pernice M, Dunn SR, Tonk L, Dove S, Domart-Coulon I, Hoppe P et al. (2014). A nanoscale secondary ion mass spectrometry study of dinoflagellate functional diversity in reef-building corals. Env Microbiol; e-pub ahead of print 30 June 2014; doi:10.1111/1462-2920.12518.

Pettay DT, Lajeunesse TC (2007). Microsatellites from clade B Symbiodinium spp. specialized for Caribbean corals in the genus Madracis. Mol Ecol Notes 7: 1271-1274.
Porter J (1976). Autotrophy, heterotrophy, and resource partitioning in Caribbean reef-building corals. Am Nat 110: 731-742.

Porter SS, Simms EL (2014). Selection for cheating across disparate environments in the legume-rhizobium mutualism. Ecol Lett 17: 1121-1129.

Prada C, Hellberg ME (2013). Long prereproductive selection and divergence by depth in a Caribbean candelabrum coral. Proc Natl Acad Sci USA 110: 3961-3966.

Prada C, McIlroy SE, Beltrán DM, Valint DJ, Ford SA, Hellberg ME et al. (2014). Cryptic diversity hides host and habitat specialization in a gorgonian-algal symbiosis. Mol Ecol 23: 3330-3340.

Prada C, Weil E, Yoshioka P. (2010). Octocoral bleaching during unusual thermal stress. Coral Reefs 29: 41-45.

Ribes M, Coma R, Gili J (1998). Heterotrophic feeding by gorgonian corals with symbiotic zooxanthella. Limnol Oceangr 43: 1170-1179.

Roffman B (1968). Patterns of oxygen exchange in some Pacific corals. Comp Biochem Physiol 27: 405-418.

Sánchez J, McFadden C, France S, Lasker H (2003). Molecular phylogenetic analyses of shallow-water Caribbean octocorals. Mar Biol 142: 975-987.

Sánchez J, Wirshing H (2005). A field key to the identification of tropical western Atlantic zooxanthellate octocorals (Octocorallia: Cnidaria). Caribb J Sci 41: 508-522.

Swanson R, Hoegh-Guldberg O (1998). Amino acid synthesis in the symbiotic sea anemone Aiptasia pulchella. Mar Biol 131: 83-93.

Thibaudeau SA (1983). The composition, form and function of spicules in octocorals. Undergraduate Thesis, Brock University.

Tremblay P, Grover R, Maguer J-F, Legendre L, Ferrier-Pages C (2012). Autotrophic carbon budget in coral tissue: a new ${ }^{13} \mathrm{C}$-based model of photosynthate translocation. J Exp Biol 215: 1384-1393.

Wainwright S, Dillon J (1969). On the orientation of sea fans (genus Gorgonia). Biol Bull 136: 130-139.

Wainwright SA (1967). Diurnal activity of hermatypic gorgonians. Nature 216: 1041-1041.

Wilkerson FP, Muscatine L (1984). Uptake and assimilation of dissolved inorganic nitrogen by a symbiotic sea anemone. Proc Natl Acad Sci Biol 221: 71-86.

Wilkinson C (1983). Net primary productivity in coral reef sponges. Science 219: 410-412.

Yellowlees D, Rees TAV, Leggat W (2008). Metabolic interactions between algal symbionts and invertebrate hosts. Plant Cell Environ 31: 679-694.

(c) (1) $\circledast$ T) This work is licensed under a Creative Commons Attribution-NonCommercial-

NoDerivs 4.0 International License. The images or other third party material in this article are included in the article's Creative Commons license, unless indicated otherwise in the credit line; if the material is not included under the Creative Commons license, users will need to obtain permission from the license holder to reproduce the material. To view a copy of this license, visit http://creativecommons.org/ licenses/by-nc-nd/4.0/ 